\title{
A NEW PROOF OF A RESULT OF LEVITZKI
}

\author{
ABRAHAM A. KLEIN
}

\begin{abstract}
A very short proof of the result which states that a nil ring of bounded index has a nonzero nilpotent ideal is given and it is shown that the same method of proof yields a more general result.
\end{abstract}

We present a new proof of the following well-known result which is attributed to Levitzki ([1, p. 374], [2]; for a proof see [3, pp. 1-2]): If a ring $R$ has a nonzero right ideal $A$ which is nil of bounded index, then $R$ has a nonzero nilpotent ideal.

An element $b \in R$ satisfying $b R b=0$ generates a nilpotent ideal, so we prove the existence of such a nonzero element.

Let $n>1$ be such that $x^{n}=0$ for all $x \in A$ and take $a \in A, a \neq 0, a^{2}=0$. It follows that $(a y)^{n-1} a=(a y+a)^{n}=0$ for all $y \in R$. Let $k>1$ be minimal for which there exists $0 \neq b \in R$ such that $(b y)^{k} b=0$ for all $y \in R$. If $k>1$, take $y_{0} \in R$ satisfying $\left(b y_{0}\right)^{k-1} b \neq 0$ and let $b_{1}=\left(b y_{0}\right)^{k-1} b$. Since $b_{1}$ is a right multiple of $b$ and $b y_{0} b_{1}=0$ we get

$$
0=\left(b_{1} z+\left(b y_{0}\right)^{k-1}\right)^{k} b=\left(b_{1} z\right)^{k-1}\left(b y_{0}\right)^{k-1} b=\left(b_{1} z\right)^{k-1} b_{1}
$$

for all $z \in R$. This contradicts the minimality of $k$, so $k=1$ and $b R b=0$.

We now show that the same arguments as before can be used to give a proof of a generalization of Levitzki's result which is given in [2]. It states that if a ring $R$ has a nonzero right ideal $A$ for which there exist $c \in R$ and a fixed $n>1$ such that $A c \neq 0$ and $x^{n} c=0$ for all $x \in R$, then $R$ has a nonzero nilpotent ideal.

We take $a \in A, a c \neq 0, a^{2} c=0$ and we get $(a c y)^{n-1} a c=(a c y+a)^{n} c=0$ for all $y \in R$. Then we let $k \geqslant 1$ be minimal for which there exists $b \in R, b c \neq 0$, $(b c y)^{k} b c=0$ for all $y \in R$. The assumption $k>1$ yields a contradiction as above, for if we take $y_{0} \in R$ satisfying $\left(b c y_{0}\right)^{k-1} b c \neq 0$ and $b_{1}=\left(b c y_{0}\right)^{k-1} b$, we get $b_{1} c \neq 0$ and

$$
0=\left(b_{1} c z+\left(b c y_{0}\right)^{k-1}\right)^{k} b c=\left(b_{1} c z\right)^{k-1}\left(b c y_{0}\right)^{k-1} b c=\left(b_{1} c z\right)^{k-1} b_{1} c
$$

for all $z \in R$.

\section{BIBLIOGRAPHY}

1. M. Chacron, Algebraic $\phi$-rings extensions of bounded index, J. Algebra 44 (1977), 370-388.

2. B. Felzenszwalb, On a result of Levitzki, Canad. Math. Bull. 21 (1978), 241-242.

3. I. N. Herstein, Topics in ring theory, Univ. of Chicago Press, Chicago, Illinois, 1969.

Department of Mathematics, Tel-Aviv University, Tel-Aviv, Israel

Current address: Department of Mathematics, University of Texas, Austin, Texas 78712

Received by the editors January 4, 1980.

1980 Mathematics Subject Classification. Primary16A22. 